\title{
A lexicographical dynamic flow model for relief operations
}

\author{
Gregorio Tirado $^{1}$, F. Javier Martín-Campo ${ }^{1}$, Begoña Vitoriano ${ }^{1}$, M. Teresa Ortuñoo ${ }^{1}$ \\ ${ }^{1}$ HUM-LOG research group, Universidad Complutense de Madrid, \\ Plaza de Ciencias 3, \\ Madrid, 28040, Spain \\ E-mail: \{gtiradod,fmart02,bvitoria,mteresa\}@ucm.es
}

Received 11 January 2013

Accepted 29 June 2013

\begin{abstract}
Emergency management is a highly relevant area of interest in operations research. Currently the area is undergoing widespread development. Furthermore, recent disasters have highlighted the importance of disaster management, in order to alleviate the suffering of vulnerable people and save lives. In this context, the problem of designing plans for the distribution of humanitarian aid according to the preferences of the decision maker is crucial. In this paper, a lexicographical dynamic flow model to solve this problem is presented, extending a previously introduced static flow model. The new model is validated in a realistic case study and a computational study is performed to compare both models, showing how they can be coordinated to improve their overall performance.
\end{abstract}

Keywords: Humanitarian Logistics, Distribution models, Multicriteria Dynamic Flow Models

\section{Introduction}

Emergency management is nowadays a high relevance area of interest that is currently being notably developed. Furthermore, recent disasters such as the January 2010 Haiti earthquake, the March 2011 disaster concatenation in Japan or the August 2012 floods in Philippines have highlighted the importance of disaster management, in order to alleviate the suffering of vulnerable people and save lives. In this direction, growing efforts are being made in the scientific community toward the development of decision aid systems and models for humanitarian logistics. Van Wassenhove and Pedraza-Martínez ${ }^{24}$ is a recent example on the use of operations research in humanitarian operations. An up-to-date review of decision aid models for disaster management and emergencies can be found in Ortuño et al. ${ }^{16}$ and
Liberatore et al. ${ }^{13}$.

One of the main problems of interest in disaster management deals with the distribution of humanitarian aid. The planning of this distribution is done along the different phases of the process (pre and post disaster). In this work we will focus on the last mile distribution, related to operating decisions on the field. This means that the transportation network, the resources allocation and demand are already defined for the operation to be developed. In this context, the aim of the problem consists of designing a set of itineraries for the vehicles to distribute the aid, meeting the conditions related to load and vehicle availability and taking into account some performance criteria.

Several versions of this problem have already been approached in the literature. The distribution problem with a single objective function has 
been approached using different kinds of models. A multi-commodity and multi-modal network flow problem considering time windows was presented by Haghani and $\mathrm{Oh}{ }^{10}$ where the objective is minimizing costs. This model was extended by Barbarosoglu and Arda ${ }^{3}$ by considering a two-stage stochastic optimization problem. Özdamar et al. ${ }^{18}$ developed a multi-period and multi-commodity network flow model minimizing the total unsatified demand. Angelis et al. ${ }^{1}$ addressed an integer linear optimization model maximizing the total demand satisfied for the World Food Programme in Angola. A mixed integer optimization model in which resource allocation and vehicle routing are taken into account together is studied in Balcik and Beamon ${ }^{2}$.

As it can be seen, there are several criteria (cost, time, unsatisfied demand, etc.) that are relevant in this context. To deal with them jointly some multicriteria models have been studied. For instance, in Viswanath and Peeta ${ }^{25}$ a multi-commodity model minimizing the total travel time and maximizing the total population covered is addressed. Tzeng et al. ${ }^{23}$ use a fuzzy multiple-objective model considering three different criteria: operation cost, total travel time and demand satisfaction. Nolz et al. ${ }^{14}$ also propose a multi-objective model including facility location with three different criteria: covering, length of tours and maximum travel time.

Attending to the dynamic nature of the problem, dynamic network flow models (which have been studied in many fields, see Cai et al. ${ }^{5}$ for a survey), have been also applied in humanitarian logistics. Fiedrich et al. ${ }^{7}$ address a dynamic model for resource allocation in case of strong earthquakes, minimizing the total number of fatalities related to different facts. Yi and Kumar ${ }^{27}$ present an ant colony optimization in two phases, one focused on the routes generation and the other one on the assignment of vehicles and commodities. Yi and Özdamar ${ }^{28}$ develop an integrated locationdistribution model for coordinating logistics support and evacuation operations in case of disaster. In both models, the objective function includes the weighted sum of the unsatisfied demand and the wounded people waiting for help. Sheu ${ }^{21}$ addresses an hybrid fuzzy clustering-optimization approach considering dynamic demand, and including a multi-objective function in which the time-varying relief demand fill rate is maximized and the time-varying distribution cost is minimized. Gonçalves ${ }^{9}$ illustrates how dynamic systems can help the humanitarian managers learn and understand the complex dynamic involved when a disaster occurs. Hamacher et al. ${ }^{11}$ present two modelling tools to predict and evaluate evacuation plans by considering a dynamic network, where the flow is maximized in a single objective function. Ben-Tal et al. ${ }^{4}$ propose a methodology based on robust optimization for risk mitigation in humanitarian relief supply chains, assigning emergency response and evacuation traffic flow in a dynamic way and considering uncertain demand.

In Ortuño et al. ${ }^{17}$ a static flow model to obtain a planning of the humanitarian aid distribution is presented, managing up to six conflicting criteria through a lexicographic goal programming model. In this paper, a dynamic flow model that builds upon that static model is presented, allowing to obtain an operation scheduling through a time horizon. The proposed model manages explicitly the movement of vehicles in the different periods of time (allowing visiting a node in several times, in opposition to the static model). Four criteria are considered in the decision process: global quantity distributed, time of operation, equity of the aid distribution, and cost. The first criterion, related to the effectiveness of the operation, is considered to be incomparably more important than the others, and thus the model is formed by two priority levels considered in a lexicographic order. Besides, several hard constraints, such as budget or resources availability, are also taken into account. A preliminary version of this model was introduced in Tirado et al. ${ }^{22}$.

From the previous review it follows that, on the one hand, the static models of the literature provide a planning for the operation but not necessarily a realistic scheduling, and on the other hand, the dynamic models of the literature do not focus on the multicriteria nature of the problem. The main gap of the state of the art filled by this paper is the proposal of a dynamic model that does provide a suitable scheduling for the operation and considers jointly attributes such as quantity, cost, time and equity of distribution 
to help the decision maker obtain solutions according to his/her preferences.

In Vitoriano et al. ${ }^{26}$, that proposes a static flow model with some additional performance measures used to better evaluate the considered criteria, the resulting static model was validated on a case study based on the 2010 Haiti earthquake. This case study will be used in the computational experience of this paper to evaluate the performance of the proposed dynamic model and compare it with its static version.

The organization of the rest of the paper is as follows. Section 2 describes the problem addressed in this work. Section 3 presents the main elements of the dynamic model. Section 4 is devoted to the different criteria to better distribute the humanitarian aid. In Section 5 a realistic case, based on the Haiti catastrophic earthquake, is studied, and the performance of the two proposed models is evaluated. Finally, Section 6 concludes and presents the main lines of future research.

\section{Problem description}

The problem addressed in this work concerns lastmile distribution in disaster relief operations. Normally, once a disaster occurs, the goods to be distributed are located in certain places (warehouses previously built on airports, ports, etc. to receive humanitarian aid coming from outside). Also, the aid is usually distributed to the population in some temporal shelters or distribution points where people go looking for help. The aim of the problem consists in designing routes for vehicles among depots and demand nodes, choosing the types of vehicles more adequate for distribution and determining the flow of the aid. The following elements compose the distribution problem:

1. Transportation Network. It is constituted by nodes, representing the places of pick-up, delivery or connection, and the main links that are characterized by distance and average velocity.

2. Goods. The information about the quantity of humanitarian aid, available or required at each node, is provided.

3. Vehicles. Several types are considered and they are characterized by capacity, average velocity, variable and fixed costs and availability in each node of the network.

4. Operation elements. The global quantity to be distributed in the operation and the budget available.

Then, the problem consists of distributing the planned amount of humanitarian aid using the available resources and infrastructures without exceeding the given budget. For this purpose, we present in this paper a dynamic flow model, building upon the one presented in Ortuño et al. ${ }^{17}$ by considering a time horizon divided in periods of time. That model was able to provide solutions based on load and vehicle flows that could be used as indications of how to proceed, but as all the static flow models, they do not provide a realistic scheduling. However, the dynamic model proposed in this paper is able to provide the decision maker with an implementable plan, by giving information about the routes of each vehicle and the time instants when each event happens.

The proposed approach is composed by two priority levels. At the first level, the goal is to distribute the amount of humanitarian aid initially planned, or if this cannot be done with the available resources, to distribute as much as possible. At the second level, once the total amount to be transported is fixed, an itinerary is designed to meet a set of goals related to other performance criteria aggregated in a weighted goal programming model. This second level can be guided by different criteria. However, the traditional logistic attributes such as cost or profit are not the most relevant in humanitarian operations. On the contrary, the time of operation or equity in the distribution, for example, take a prominent role, as it will be considered in our approach.

It is important to remark that the two phases of the model are considered in a lexicographic order, since delivering all aid, in order to reach the maximum amount of people in need, is incomparably preferred as meeting any other goals related to cost, 
time or equity and no trade-off is possible when dealing with the amount of aid delivered. Besides, it must be taken into account that all hard constraints, as for example the ones related to the maximum budget or resource availability, must be met by any feasible solution in any case, so there is not possible trade-off when dealing with them.

\section{Dynamic Flow Model}

The dynamic distribution model presented in this paper is based on two dynamic network flow models, one for the load of humanitarian aid and another one for the vehicles, that are closely related. A complete description of these models, together with the constraints related to the resources available to perform the operation, are presented in the following. A preliminary version of these models was presented in Tirado et al. ${ }^{22}$.

\subsection{Sets and parameters}

A) Main sets and indices.

$(N, A)$ : transport network, where $N$ and $A$ denote the set of nodes and arcs, respectively.

$T$ : planning horizon; the mission can be developed through periods $\{1, \ldots, T\}$.

$i, i^{\prime}:$ indices to refer nodes $i, i^{\prime} \in N$.

$t, t^{\prime}:$ indices to refer time periods $t, t^{\prime} \in\{1, \ldots, T\}$.

$V$ : set of vehicle types, defined by their characteristics.

$j, j^{\prime}:$ indices to refer vehicle types, $j, j^{\prime} \in V$.

B) Parameters related to the network.

dist $_{i i^{\prime}}$ : length of $\operatorname{arc}\left(i, i^{\prime}\right) \in A$.

dem $_{i}$ : demand at node $i \in N$, in units of load.

$q a v_{i}:$ supply at node $i \in N$, in units of load.

C) Parameters related to vehicles. (Several types are considered). $\operatorname{cap}_{j}$ : capacity of vehicle type $j \in V$, in units of load.

$v a v_{j i}$ : number of available vehicles of type $j \in V$ at node $i \in N$.

$c f_{i i^{\prime} j}$ : fixed cost of travelling through arc $\left(i, i^{\prime}\right) \in A$ with a vehicle of type $j \in V$, per unit of length.

$c v_{i i^{\prime} j}$ : cost of travelling through arc $\left(i, i^{\prime}\right) \in A$ with a vehicle of type $j \in V$, per unit of length and load.

$t r_{i i^{\prime} j}$ : periods of time needed to travel from $i \in N$ to $i^{\prime} \in N$ with a vehicle of type $j \in V$.

D) Parameters related to the operation.

$q$ : amount of aid desired to be distributed in the operation.

$b$ : budget available to perform the operation.

For simplicity, in the following it will be assumed that, unless otherwise stated, indices $i, i^{\prime}, j, j^{\prime}, t, t^{\prime}$ take values in the sets where they are defined, so that only existing arcs and vehicle types compatible with them are considered in the definition of the constraints.

\subsection{Variables}

$Q L_{i i^{\prime} j t}$ : load carried from $i$ to $i^{\prime}$ using vehicle type $j$ and starting in period $t$.

$Q_{i t}$ : load staying (stored or received) at node $i$ in period $t$.

$N L_{i i^{\prime} j t}$ : number of vehicles of type $j$ which start travelling from $i$ to $i^{\prime}$ in period $t$.

$N_{i j t}$ : number of vehicles of type $j$ that are at node $i$ at the beginning of period $t$.

Note that the load and vehicles that are at each node at the end of the operation are given by the variables $Q_{i T}$ and $N_{i j T}$, that represent, respectively, the amount of humanitarian aid and the number of vehicles of type $j$ that stay at node $i$ when all itineraries have been completed. 


\subsection{Structural Model}

$$
\begin{gathered}
\sum_{i^{\prime}} \sum_{j} \sum_{t^{\prime} \leqslant t-t r_{i^{\prime} \prime} j} Q L_{i^{\prime} i j t^{\prime}}+q a v_{i}= \\
\sum_{i^{\prime}} \sum_{j} \sum_{t^{\prime} \leqslant t} Q L_{i i^{\prime} j t^{\prime}}+Q_{i t} \quad \forall i, t \\
\sum_{i} Q_{i T}=\sum_{i} q a v_{i} \\
\sum_{i^{\prime}} \sum_{t^{\prime} \leqslant t-t r_{i^{\prime} i j}} N L_{i^{\prime} i j t^{\prime}}+v a v_{j i}= \\
\sum_{i^{\prime}} \sum_{t^{\prime} \leqslant t} N L_{i i^{\prime} j t^{\prime}}+N_{i j t} \quad \forall i, j, t \\
\sum_{i} v a v_{j i}=\sum_{i} N_{i j T} \quad \forall j \\
Q L_{i i^{\prime} j t} \leqslant c a p_{j} N L_{i i^{\prime} j t} \quad \forall i, i^{\prime}, j, t \\
\sum_{i, i^{\prime}, j, t} \operatorname{dist}_{i i^{\prime}}\left(2 c f_{i i^{\prime} j} N L_{i i^{\prime} j t}+c v_{i i^{\prime} j} Q L_{i i^{\prime} j t}\right) \\
C O S T \leqslant b \\
\sum_{i / d e m_{i}>0} Q_{i T} \leqslant q \\
Q L_{i i^{\prime} j t}, Q_{i t} \geqslant 0 \quad \forall i, i^{\prime}, j, t \\
N L_{i i^{\prime} j t}, N_{i j t} \geqslant 0 \text { and integer } \forall i, i^{\prime}, j, t
\end{gathered}
$$

Conditions (1) are the dynamic load flow balance equations at each node and time period. Constraint (2) ensures that the total load staying at the nodes at the end of the operation is the available load. Conditions (3) are, analogously, the dynamic flow balance equations for the vehicles, while conditions (4) ensure that only the vehicles that are available are used for transportation. Constraints (5) impose that the maximum capacity of vehicles is not exceeded. Expression for cost is introduced in condition (6) and the maximum available budget in condition (7). Constraint (8) ensures that the maximum amount of load planned in the operation is not exceeded. Ideally, it should be an equality, but it could be unfeasible to deliver all planned aid, introducing it as a goal (this issue will be clarified in the next section). Finally, constraints (9)-(10) state the domain of the variables.
Please note that, in the plans given by the static model, all flow of aid leaving one node is supposed to be sent at the same time and thus two vehicle departures from the same node cannot take place at different moments; however, as it can be observed in the structural model, the dynamic model does not suffer from this limitation, because it allows some vehicles to depart from a given node before or after some other vehicles did (see variables $N L_{i i^{\prime} j t}$ ), providing a particular schedule for each vehicle.

\section{Attributes and criteria aggregation}

Criteria concerning the amount of goods distributed, time of operation, the equity of the distribution and the cost of operation will be considered. For each of these attributes a target will be defined and the goal of achieving this target will be included.

The global amount of aid desired to be distributed has been defined previously in condition (8), where the target is included as the parameter $q$.

COST is calculated by adding both fixed and variable costs as stated in equation (6). As condition (7) stated before, it is bounded by the available budget, but additionally, a desired target could be defined.

For equity, a worst case philosophy is applied, defining it as the most unsatisfied demand:

$$
D X \geqslant 1-\frac{Q_{i T}}{\operatorname{dem}_{i}} \quad \forall i / \operatorname{dem}_{i}>0
$$

$D X$ is intended to measure how equitable the distribution plan is, by calculating the largest proportion of unsatisfied demand among the nodes. It is a real number between 0 and 1: a distribution plan in which the demand of all nodes is completely fulfilled is associated to 0, while any solution in which at least one demand node receives nothing is associated to 1 . Note that the ideal value $D X=0$ is only achievable if the offer and the quantity finally distributed in the operation are not smaller than the demand. Otherwise, the most equitable itinerary is obtained if $D X=1-\frac{\text { aid to be delivered }}{\text { total demand }}$, meaning that exactly the same proportion of aid is transported to each node. A target $t g_{D X}$ will be defined by the decision maker. 
Two measures $T_{1}$ and $T_{2}$ dealing with the operation time are introduced:

$$
\begin{array}{ll}
T_{1} \geqslant t \cdot B Q_{t} & \forall t>t g_{T 1} \\
T_{2}=\sum_{t>t g_{T 2}}\left(t-t g_{T 2}\right)^{2} \cdot B Q_{t} &
\end{array}
$$

where $B Q_{t}$ is a binary variable being 1 if all load has been delivered in period $t$ and 0 otherwise, and $t g_{T 1}$ and $t_{T 2}$ are the targets for $T_{1}$ and $T_{2}$, respectively.

$T_{1}$ and $T_{2}$ are time measures defined so that they are equal to 0 if the corresponding time target $\left(\operatorname{tg}_{T 1}\right.$ or $\left.t g_{T 2}\right)$ is achieved and they are positive otherwise. Both variables will be used to measure the deviation from the time targets, with a different purpose. If the target $\operatorname{tg}_{T 1}$ is not achieved, $T_{1}$ represents the number of time periods required to complete the operation, as stated by constraints (12). Hence, $T_{1}$ grows linearly with the number of time periods used. However, we realized that it could be useful to consider some other variable with a faster increase with the time of the operation, in order to add higher penalties to long operations. For this purpose, we defined $T_{2}$ as stated in equation (13), so that it has a quadratic increase on the number of time periods used if the time target $\operatorname{tg}_{T 2}$ is not achieved.

In order to guarantee that the binary variables $B Q_{t}$ are defined correctly, constraints (14) to (18) are added to the model:

$$
\begin{aligned}
B Q_{t+1} & \leqslant B Q_{t} & & \forall t \\
N_{i j t}-N_{i j T} & \leqslant m_{j} \cdot B Q_{t} & & \forall i, j, t \\
N_{i j T}-N_{i j t} & \leqslant m_{j} \cdot B Q_{t} & & \forall i, j, t \\
\sum_{i} \sum_{i^{\prime}} N L_{i i^{\prime} j t} & \leqslant m_{j} \cdot B Q_{t} & & \forall j, t \\
B Q_{t} & \in\{0,1\} & & \forall t
\end{aligned}
$$

where $m_{j}=\sum_{i} v a v_{j i}$ for each $j$, that are bounds representing the total number of vehicles of each type $j$ available in the network.

Notice that $B Q_{t}$ is equal to 0 only if $N_{i j t}=N_{i j T}$ for all $i, j$ (see equations (15)-(16)), i.e., when all vehicles have reached their final destination at time period $t$. Note also that if $B Q_{t}=0$ for a given $t$ (representing that the operation is finished at time period $t$ ), it holds that $B Q_{t^{\prime}}=0$ for all $t^{\prime}>t$, as implied by equation (14).

The proposed attributes are aggregated using a goal programming scheme, based on a satisfying philosophy. As indicated earlier, goal programming (see, for example, Charnes and Cooper ${ }^{6}$ and Romero ${ }^{20}$ ) is based on the achievement of a set of goals, more than on the optimization of the performance measures. The resulting goal programming model requires a set of additional conditions named goal constraints (20)-(24), used to measure the deviations from the targets.

$$
\begin{aligned}
& \sum_{i \mid d e m_{i}>0} Q_{i T}+D V_{Q}=q \\
& C O S T-D V_{C} \leqslant t g_{C} \\
& D X-D V_{D} \leqslant t g_{D} \\
& T_{1}-D V_{T 1} \leqslant \operatorname{tg}_{T 1} \\
& T_{2}-D V_{T 2} \leqslant t g_{T 2} \\
& \operatorname{COST}, T_{1}, T_{2}, D X, D V_{C}, D V_{D}, D V_{T 1}, D V_{T 2} \geqslant 0
\end{aligned}
$$

Parameter $q$ is the target for the amount of aid to be distributed, and coefficients $t g_{C}, t g_{D}, t g_{T 1}$ and $t g_{T 2}$ represent the targets defined by the decision maker for the attributes related to COST, $D X, T_{1}$ and $T_{2}$, respectively. Non-negative variables $D V_{Q}, D V_{C}, D V_{D}$, $D V_{T 1}, D V_{T 2}$ are the unwanted deviations from the given targets in the solution.

The problem is solved by lexicographical goal programming, corresponding to two priority levels. The primary goal is distributing the planned amount of goods, or if unfeasible, delivering as much as possible, understood as a criterion of effectiveness. It is considered that there is not possible trade-off between this criterion and other criteria such as time, cost or equity. Then, the first priority level is defined as reaching the goal of the quantity to be distributed, and once the value of the deviation variable of equation (19) is fixed, the second level deals with the rest of goals.

Level 1 is thus defined by (25), where the deviation of delivered aid with respect to the planned amount of the operation is minimized.

$$
\min D V_{Q} \text { s.t. (1) to (10),(19) }
$$


Once level 1 is solved and the optimal value of $D V_{Q}^{*}$ is obtained, constraint (19) must be redefined to ensure that this amount of humanitarian aid is also distributed in the second level. This is done by replacing constraint (19) by constraint (26).

$$
\sum_{i \mid d e m_{i}>0} Q_{i T}+D V_{Q}^{*}=q
$$

Hence, level 2 is defined by (27), where, once the amount of aid to be delivered is fixed, the sum of weighted deviations from the other targets is minimized.

$$
\min \sum_{v} \frac{\alpha_{v}}{t_{v}} D V_{v} \text { s.t. (1) to (18), (20) to (24), (26) }
$$

Index $v$ represents the attributes considered for the second level of priority (COST, $\left.T_{1}, T_{2}, D X\right), \alpha_{v}$ is the weight given by the decision maker to attribute $v$ and $t_{v}$ is its target (in order to manage together deviations measured in different units, the variables are divided by their targets).

For technical purposes (obtaining an initial integer solution for the second level), equations (11) to (24) will also be included in the first level, but note that, since the deviation variables are not included in the objective function, attributes related to cost, time or equity do not affect the final solution until level 2.

\section{Case Study}

The aim of this section is to compare the proposed dynamic model with the static one proposed in Ortuño et al. ${ }^{17}$, and also to study how they can be coordinated to improve their performance. This is done by using a realistic base case study to illustrate how the models could be used in practice (Section 5.1), and also a testbed created from that base case, that allows for a more comprehensive computational study (Section 5.2).

\subsection{Base case study}

The static and the dynamic models proposed have been tested and compared on a case study built on the Haiti catastrophic earthquake of January 12th,
2010. It is based on the planning of an operation of humanitarian aid distribution in Port-au-Prince, Haiti's capital, and its surroundings. The main data of the base case study have been mostly estimated from the information contained at the web sites OCHA ${ }^{15}$ and Redhum.org ${ }^{19}$.

The transportation network consists of 24 nodes and 42 available links between locations. There are 9 settlements demanding a total of 250 tons of load, 3 depots (the port with 60 tons of available aid, the airport with 80 tons, and Jimaní, border city of Dominican Republic, with 140 tons) and 12 intermediate nodes.

In Figure 1 a map of the region is presented, showing the network nodes (depots labeled 1-3, demanding nodes labeled by $10,12,13,16,17,18$, $20,21,22$, and the rest for intermediate nodes) and all available links. The demand of the demanding nodes is, respectively, 30, 40, 30, 30, 10, 30, 40, 20, 20 tons.

The thickness of each link is related to its quality (depending on if it is an unpaved road, a highway, etc.), determining the maximum speed of the vehicles travelling through them (through a thicker link vehicles can travel faster).

We have considered the use of 3 types of vehicles available for transportation, that are parked at the depots and at a few intermediate nodes.

The final cost of the operation is given in dollars and the time of response in minutes (not including loading and unloading operations). Finally, the planning operation consists of delivering 150 tons of humanitarian aid with a budget of 80,000 dollars.

For additional details on this base case study we refer the reader to Vitoriano et al. ${ }^{26}$, where the same base case study was also used to test the static flow model mentioned earlier.

Both the static and the dynamic model were implemented in GAMS ${ }^{8}$ and solved using CPLEX ${ }^{12}$ in parallel mode as optimizer.

For this case study the duration of each time period is chosen to be one minute. Besides, as the time horizon is related to the total duration of the operation, the operation time given by the static flow model, increased by $10 \%$, has been used to approximate the time horizon. 


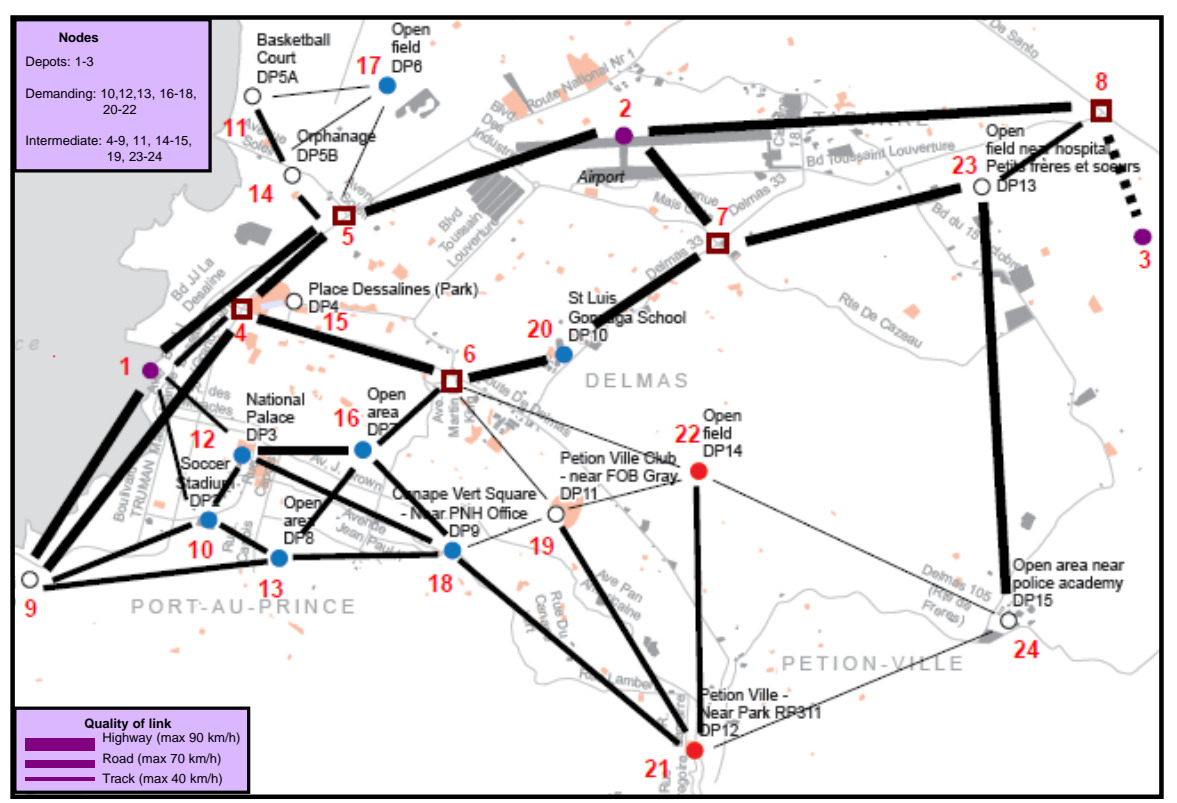

Fig. 1. Transport network at Port-au-Prince (OCHA, 31

January 2010)

The targets proposed for the second level goals, chosen in a very optimistic way, are $40,000 \$$ for the cost, 30 minutes for the operation time and 0.4 for the equity. The corresponding weights, also for the second level goals, are the following:

- Static model: $\alpha_{C}=1, \alpha_{D}=2, \alpha_{T}=2$.

- Dynamic model: $\alpha_{C}=1, \alpha_{D}=2, \alpha_{T 1}=\alpha_{T 2}=1$.

According to these weights, all attributes are taken into account, but the equity of the distribution and the time of response are given a higher importance than the cost of the operation. Note also that, since the static model only uses one time measure $T$ and the dynamic model uses two time measures $T_{1}$ and $T_{2}$, the weight related to time, that is $\alpha_{T}=2$, is divided in two parts in the dynamic model, so that $\alpha_{T 1}+\alpha_{T 2}=1+1=\alpha_{T}$.

Figures 2 and 3 illustrate the solutions provided by the static flow model and the dynamic model, re- spectively, when applied to the case study described above. In both cases, goals related to the quantity to be distributed are reached.

In Figures 2 and 3 it can be observed that the itineraries given by both models are quite similar, differing in only a few links and delivering the same amount of humanitarian aid to each demand node. Furthermore, the values of the attributes of both solutions are also quite alike: the cost of the solution provided by the dynamic model is just $0.35 \%$ higher than the cost of the solution provided by the static one, while the operation time is $6.67 \%$ higher. Both the cost and the operation time are higher in the dynamic model due to the discretization of the time in periods; however, the itineraries given by the dynamic model are much closer to a real operation, and the same node could be visited at different times. Besides, since the same load is delivered to each node, the equity is identical in both cases. 


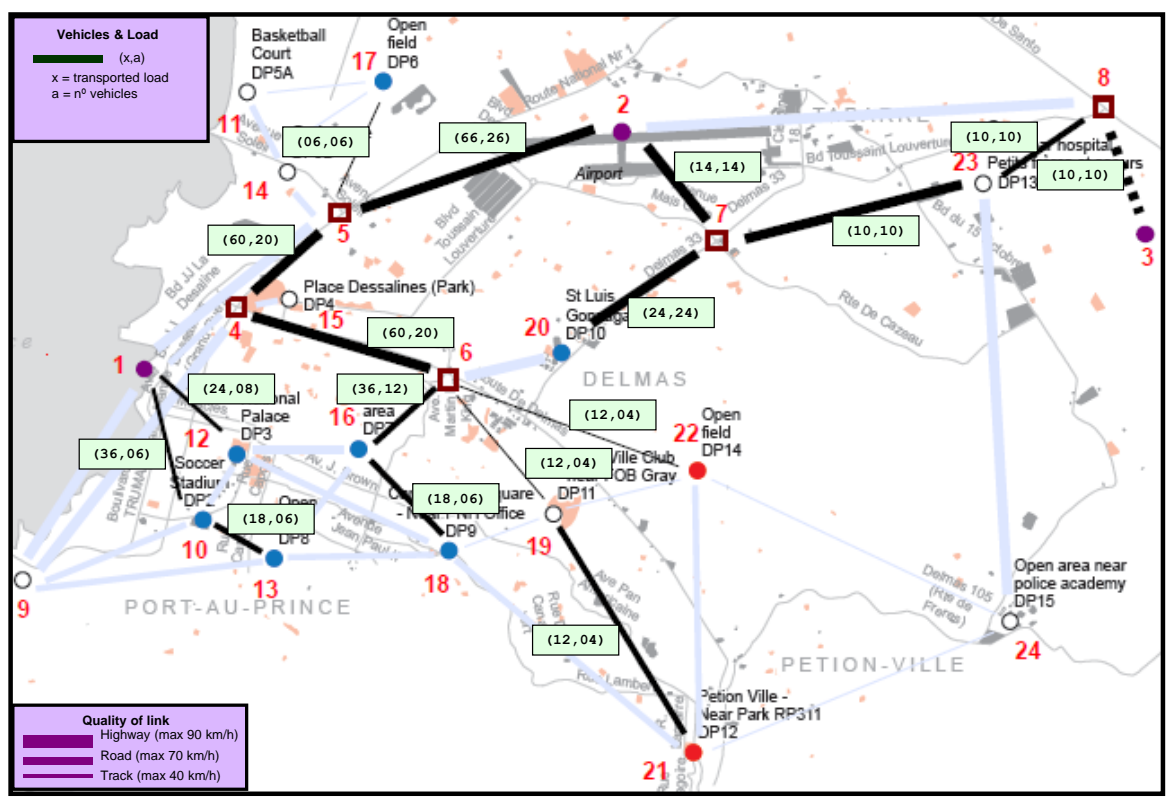

Fig. 2. Itineraries given by the static model on the original case study

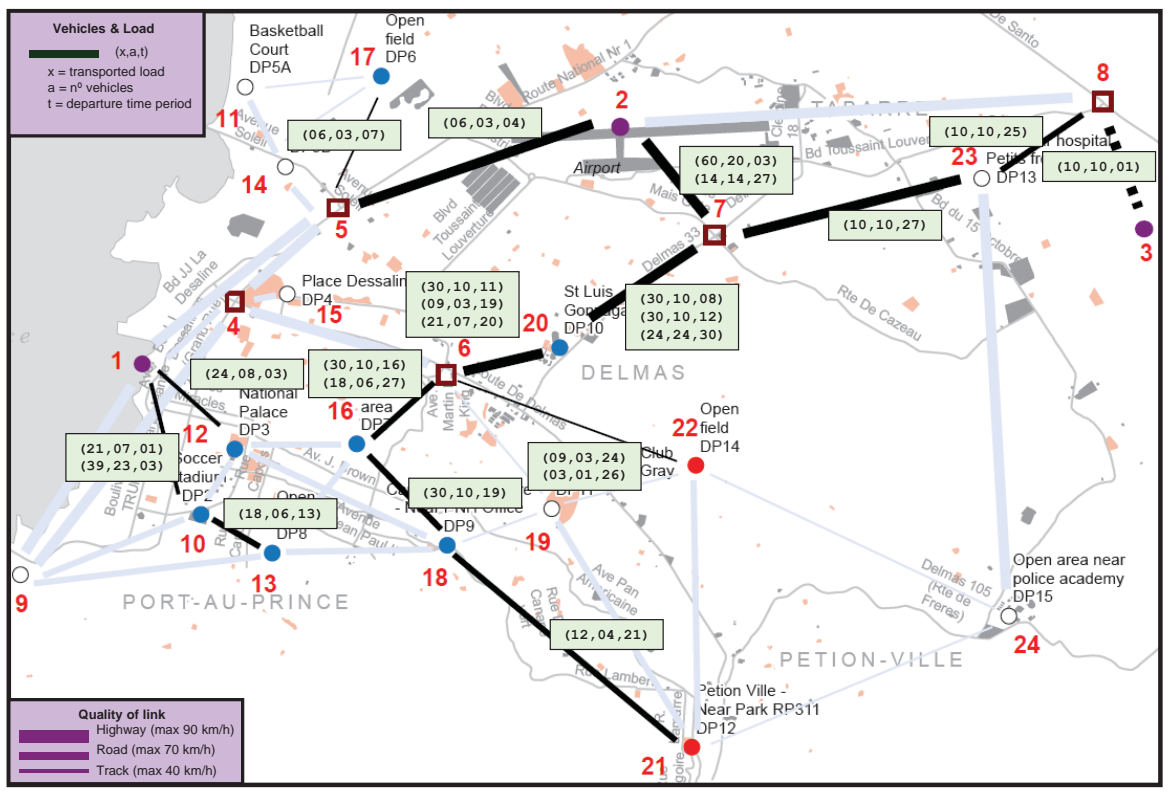

Fig. 3. Itineraries given by the dynamic model on the original case study 
This suggests that the static model approximates quite well the dynamic one. Note that for the dynamic model it is crucial to estimate properly the maximum number of time periods that are going to be used because model dimensions increase dramatically with this parameter. However, if they are too few, it may not exist any feasible solution, or the available time horizon would be so restricting that several criteria could not have any implication. As a result, we have decided to use the information of the solution provided by the static model concerning the operation time to evaluate the number of time periods to be used in the dynamic model.

\subsection{Computational study}

Starting from the initial base case study introduced in the previous section, we have created 8 more test instances by changing the maximum speed of the vehicles and on the links, with the aim of making the distribution times of the operations change so that the new testbed could be used to compare the performance of the static and the dynamic model in more detail. This has been done by reducing the speeds to $20 \%, 30 \%, \ldots, 90 \%$ of the original values of the base case, obtaining 8 additional cases.

This testbed has been created in order to study computationally the performance of the dynamic model, because the slower the speed the higher the operation time, and consequently, the higher the number of time periods needed. Notice that the dimensions of the static model are independent of the travel and operation times and it can be solved quite fast in any case. However, the dynamic model requires a higher number of time periods if the operation time is larger, increasing the model dimensions and making it more difficult to solve. A solution to this problem could be addressed by increasing the length of the time periods, but this would lead to higher discretization errors. Besides, for this computational study this has not been done also to facilitate the comparison between both models.

The values of the attributes of the solutions provided by the two models on the 9 considered cases are given in Table 1. The case considered is displayed in the first column and the information regarding cost, time of response and equity is given in columns 2-3, 4-5 and 6-7, respectively. On each of these groups, the first column shows the value of the corresponding attribute in the solution provided by the static flow model and the second column shows the percentage increase observed in the solution provided by the dynamic model.

Table 1. Comparison of the attributes on the two models

\begin{tabular}{|c|c|c|c|c|c|c|}
\hline se & & & & & & \\
\hline 20 & 46391 & 53.79 & 139.76 & 3.75 & 0.4 & 10 \\
\hline 30 & 46391 & 0.35 & 93.17 & 3.04 & 0.4 & \\
\hline 40 & 46391 & 0.35 & 69.88 & 4.46 & 0. & \\
\hline 50 & 46391 & 2.94 & 55.90 & 3.76 & 0. & \\
\hline 60 & 46391 & 0.35 & 46.59 & 7.32 & 0. & \\
\hline 70 & 46391 & 0.35 & 39.93 & 5.18 & 0.4 & \\
\hline 80 & 46391 & 0.35 & 34.94 & 8.76 & 0. & \\
\hline 90 & 46391 & 0.35 & 31.06 & 9.47 & 0.4 & \\
\hline 100 & 46391 & 0.35 & 30.00 & 6.67 & 0.4 & \\
\hline
\end{tabular}

On the one hand, it can be observed that the first case (all speeds are reduced to only $20 \%$ of the original values) behaves differently than the others. This is so because the dynamic model could not be solved to optimality in less than half an hour of running time, and thus the process was ended before obtaining the optimal solution. As a consequence, the solution provided by the solver is the initial integer solution found in level 1 , in which cost, time or equity were not taken into account. Then, the two solutions given by the static and the dynamic model are significantly different: the costs differ in more than $50 \%$ and the itinerary given by the dynamic model is much less equitable, leaving some nodes unvisited.

On the other hand, the solutions provided by the two models on the rest of the cases are notably similar, delivering the same amount of aid to each node (see the last column) with very similar costs (see column 3) and in a total time with small differences (between 3\% and 10\%, see column 5). This small increase of the cost and time of response on the dynamic model is due to the discretization of time, needed to divide the time horizon in periods. We observed that the designed schedules allow multiple departures from each node, reducing the arrival time at some demand nodes and thus providing a fastest response to some of the recipients, even though the longest path, determining the operation time, is indeed the same for both models. Please note also that 
solving the models using a certain tolerance may also produce solutions that are slightly different, as it happens for example in the case of 50\% speed, in which the cost is slightly higher than in the other cases.

The running times (in seconds) required to solve each model on each test case are shown in columns 2 and 3 of Table 2, together with the maximum number of periods defined on the dynamic model on each case (column 4) and the dimensions of the model: number of rows (column 5), total number of variables (column 6) and number of discrete variables (last column). The number of time periods to activate in the dynamic model on each case, as indicated before, has been decided by using the information given by the static flow model, that is much faster to solve. Hence, for the $20 \%$ speed case, since the operation time given by the static flow model was 139.76 , we decided to activate 145 time periods; analogously, an operation time of 93.17 on the $30 \%$ speed case led to the use of 102 periods, a time of 69.88 to 76 periods and times smaller than 56 to a fixed value of 61 periods for all remaining test cases.

Table 2. Complexity of the models

\begin{tabular}{r|rr|r|rrr} 
Case & t. s. & t. d. & active & rows & vars. & disc. \\
\hline $\mathbf{2 0}$ & 2.7 & $>1800.0$ & 145 & 72014 & 86973 & 36510 \\
$\mathbf{3 0}$ & 2.0 & 335.5 & 102 & 50686 & 61217 & 25675 \\
$\mathbf{4 0}$ & 1.9 & 169.9 & 76 & 37790 & 45643 & 19123 \\
$\mathbf{5 0}$ & 2.0 & 151.8 & 61 & 30350 & 36658 & 15343 \\
$\mathbf{6 0}$ & 1.9 & 332.4 & 61 & 30350 & 36658 & 15343 \\
$\mathbf{7 0}$ & 2.1 & 404.0 & 61 & 30350 & 36658 & 15343 \\
$\mathbf{8 0}$ & 2.1 & 223.1 & 61 & 30350 & 36658 & 15343 \\
$\mathbf{9 0}$ & 1.9 & 148.2 & 61 & 30350 & 36658 & 15343 \\
$\mathbf{1 0 0}$ & 2.1 & 143.2 & 61 & 30350 & 36658 & 15343
\end{tabular}

Table 2 shows clearly how, in all cases, the running time required to solve the dynamic model is significantly higher than the one to solve the static flow model, specially in the $20 \%$ speed case (see first row), which does not reach optimality in less than half an hour, in comparison with less than 3 seconds for the static one. Concerning the complexity of the model (see last 3 columns), it can be observed how it is directly related to the number of time periods that are active, highlighting again the importance of finding good estimates for the maximum number of time periods to use.
One of the main conclusions of this computational study is the close relation between the values of the criteria obtained by both models, and especially for the operation times. This relation supports the strategy of solving the model in a coordinated way, obtaining the time horizon to be included in the dynamic model from the static one.

\section{Conclusions}

A dynamic flow model for solving the aid distribution problem in a disaster situation considering multiple criteria has been presented in this paper. The model provides a realistic schedule for the distribution of humanitarian aid taking into account goals related to quantity to be distributed (effectiveness), cost, time and equity. This model builds upon the static model introduced in Ortuño et al. ${ }^{17}$ by considering a time horizon divided in periods of time that allows the model to be much closer to the operation scheduling.

The approach chosen to deal with these criteria is lexicographical goal programming with two priority levels. The first one is to reach the goal of distributing the planned amount of humanitarian aid with the available resources and infrastructures (not allowing tradeoff with other performance criteria), providing in addition an initial integer feasible solution in a very short computational time. Once the amount of aid to be distributed is fixed, the second level is intended to find a distribution scheduling meeting a set of goals related to cost, operation time and equity of the distribution.

The second level takes significantly longer to be solved, being crucial to estimate properly the number of time periods to be used, so that the solution is not restricted but the model is not unnecessarily complex to solve. The static flow model showed to be very helpful in this task, because it provides good estimates of the operation times and it can be solved very fast. This shows that the coordination of the static and the dynamic model improves significantly their performance.

Summarising, the main contributions of this paper are as follows: (1) The introduction of a dynamic flow model providing a suitable scheduling for the 
aid distribution through a time horizon, dealing with four criteria (total quantity distributed, time, equity and cost) in a lexicographical goal programming scheme. (2) The validation of the proposed approach in a realistic case study based on the Haiti 2010 earthquake. (3) The comparison between the proposed dynamic model and the static one presented in Ortuño et al. ${ }^{17}$, also based on the Haiti case study. (4) The computational experiments which suggest the strategy of using the static flow model to estimate the number of time periods to be used by the dynamic model. This shows that the coordination of both models improves significantly their performance.

Future work will be focused on the comparison of both models with other case studies, the coordination of both models to obtain a better approximation of the time horizon, the inclusion of other performance criteria such as those ones considered in Vitoriano et al. ${ }^{26}$, and the inclusion of other time measures in order to deliver goods to each demand node as soon as possible. Finally, a decision aid system should be developed, ready to be used by any organization involved into humanitarian relief operations.

\section{Acknowledgments}

This work has been supported by project TIN201232482 from the Government of Spain. We would also like to thank two anonymous referees for their valuable suggestions, that helped us improve the paper significantly.

\section{References}

1. V. D. Angelis, M. Mecoli, C. Nikoi, and G. Storchi. Multiperiod integrated routing and scheduling of World Food Programme cargo planes in Angola. Comput. Oper. Res., 34(6):1601-1615, 2007.

2. B. Balcik and B. M. Beamon. Facility location in humanitarian relief. Int. J. Logist. Res. Appl., 11(2):101121, 2008.

3. G. Barbarosoglu and Y. Arda. A two-stage stochastic programming framework for transportation planning in disaster response. J. Oper. Res. Soc., pages 43-53, 2004.
4. A. Ben-Tal, B. D. Chung, S. R. Mandala, and T. Yao. Robust optimization for emergency logistics planning: risk mitigation in humanitarian relief supply chains. Transp. Res. Part B, 45(8):1177-1189, 2011.

5. X. Cai, D. Sha, and C. Wong. Time-varying network optimization. Springer, New York, 2007.

6. A. Charnes and V.W. Cooper. Management Models and Industrial Applications of Linear Programming. Wiley, New York, 1961.

7. F. Fiedrich, F. Gehbauer, and U. Rickers. Optimized resource allocation for emergency response after earthquake disasters. Saf. Sci., pages 41-57, 2000.

8. GAMS Development Corporation. GAMS - A User's Guide. 2010.

9. P. Gonçalves. System dynamics modeling of humanitarian relief operations. MIT Sloan Research Paper No. 4704-08, 2008.

10. A. Haghani and S.C. Oh. Formulation and solution of a multi-commodity, multi-modal network flow model for disaster relief operations. Transp. Res. Part A, pages 231-250, 1996.

11. W. Hamacher, S. Heller, and B. Rupp. Flow location (flowloc) problems: dynamic network flows and location models for evacuation planning. Ann. of Oper. Res., 2011.

12. IBM ILOG. User's Manual for CPLEX 12.4. 2012.

13. F. Liberatore, C. Pizarro, C. Simón, B. Vitoriano, and M.T. Ortuño. Decision Aid Models for Disaster Management and Emergencies, chapter Uncertainty in $\mathrm{Hu}-$ manitarian Logistics for Disaster Management. Atlantis Press, 2013.

14. P. Nolz, K. Doerner, W. Gutjahr, and R. Hartl. A bi-objective metaheuristic for disaster relief operation planning. In C. Coello, C. Dhaenens, and L. Jourdan, editors, Advances in Multi-Objective Nature Inspired Computing, volume 272 of Studies in Computational Intelligence, pages 167-187. Springer, Berlin Heidelberg, 2010.

15. OCHA. Ocha: Office for the coordination of humanitarian affairs. http://ochaonline.un.org/ [date accesed January $8^{\text {th }}$ 2013], 2013.

16. M.T. Ortuño, P. Cristobal, J.M. Ferrer, F.J. MartínCampo, S. Muñoz, G. Tirado, and B. Vitoriano. Decision Aid Models for Disaster Management and Emergencies, chapter Decision Aid Models and Systems for Humanitarian Logistics. A Survey. Atlantis Press, 2013.

17. M.T. Ortuño, G. Tirado, and B. Vitoriano. A lexicographical goal programming based decision support system for logistics of Humanitarian Aid. TOP, 19:464-479, 2011.

18. L. Özdamar, E. Ekinci, and B. Kucukyazici. Emergency logistics planning in natural disasters. Ann. of Oper. Res., pages 217-245, 2004.

19. Redhum.org. Red de información hu- 
manitaria para américa latina y el caribe. http://www.redhum.org/mapas.php [date accesed January $8^{\text {th }}$ 2013], 2013.

20. C. Romero. Handbook of Critical Issues in Goal Programming. Pergamon Press, Oxford, 1991.

21. J. B. Sheu. An emergency logistics distribution approach for quick response to urgent relief demand in disasters. Transp. Res. Part E, 43(6):687-709, 2007.

22. G. Tirado, B. Vitoriano, and M.T. Ortuño. A multicriteria dynamic flow model for relief operations. In World Scientific Proceedings Series on Computer Engineering and Information Science 7, pages 9-14, 2012.

23. G. H. Tzeng, H. J. Cheng, and T. D. Huang. Multiobjective optimal planning for designing relief delivery systems. Transp. Res. Part E, 43(6):673-686, 2007.

24. Luk. N. Van Wassenhove and Alfonso. J. Pe- draza Martinez. Using OR to adapt supply chain management best practices to humanitarian logistics. Int. Trans. Oper. Res., 19:307-322, 2012.

25. K. Viswanath and S. Peeta. Multicommodity maximal covering network design problem for planning critical routes for earthquake response. Transp. Res. Rec.: J. Transp. Res. Board, pages 1-10, 2007.

26. B. Vitoriano, M. T. Ortuño, G. Tirado, and J. Montero. A multi-criteria optimization model for humanitarian aid distribution. J. Glob. Optim., 51(2):189208, 2011.

27. W. Yi and A. Kumar. Ant colony optimization for disaster relief operations. Transp. Res. Part E, 43(6):660-672, 2007.

28. W. Yi and L. Özdamar. A dynamic logistics coordination model for evacuation and support in disaster response activities. Eur. J. Oper. Res., 179:1177-1193, 2007. 